\section{La temprana}

\section{descentralización de los}

servicios de salud en la

Argentina: la construcción del sistema sanitario en Córdoba, 1930-1955

\section{Early decentralization of health services in Argentina: the construction of the health system in Córdoba, 1930-1955}

María José Ortiz Bergia

Profesora, Universidad Nacional de Córdoba; investigadora, CONICET. Miguel C. del Corro 308 5000 - Córdoba - Argentina ortizbergia.mj@gmail.com

Recebido para publicação em dezembro de 2012. Aprovado para publicação setembro de 2013.

http://dx.doi.org/10.1590/S0104-59702015005000005
ORTIZ BERGIA, María José. La temprana descentralización de los servicios de salud en la Argentina: la construcción del sistema sanitario en Córdoba, 1930-1955. História, Ciências, Saúde - Manguinhos, Rio de Janeiro, v.22, n.2, abr.-jun. 2015, p.559-575.

\section{Resumen}

Este trabajo analiza el proceso de construcción del sistema público de salud argentino, poniendo en evidencia las limitaciones que se produjeron en el proyecto de nacionalización de las políticas sanitarias en la postguerra y el papel central que tuvieron las jurisdicciones subnacionales, provincializando la provisión de los servicios. Más precisamente se visibiliza cómo, en el segundo cuarto del siglo XX, la ampliación de los servicios de salud en algunas provincias fue principalmente el resultado de la acción de las reparticiones locales antes que nacionales. Con el propósito de dilucidar mejor ese proceso, estudiamos la trayectoria de los dispositivos sanitarios públicos en la provincia de Córdoba entre 1930 y 1955.

Palabras claves: política social; políticas públicas de salud; salud pública/ descentralización; Argentina.

\section{Abstract}

This paper analyzes the process of construction of the Argentine public health system, highlighting the limitations that occurred in the proposed nationalization of health policy in the postwar period and the central role played by subnational jurisdictions, making the provision of services rendered on a provincial basis. More precisely, in this respect it is seen how the expansion of health services in some provinces shows us how, in the second quarter of the twentieth century, it was primarily the result of the action of local rather than national departments. In order to better elucidate this process, the trajectory of public healthcare facilities in the province of Córdoba between 1930 and 1955 was studied.

Keywords: public policy; health public policy; public health/decentralization; Argentina. 
$\mathrm{E}^{\mathrm{n}}$ n el segundo cuarto del siglo XX, se produjeron profundas transformaciones en los aparatos estatales dedicados a impulsar políticas sociales en la Argentina, con la ampliación de sus prestaciones, el alcance de sus intervenciones sobre la población y la modificación de sus instrumentos burocráticos de acción. En ese contexto, una de las áreas de mayor actuación del Estado dentro del campo de lo social fue la salud que incrementó su centralidad dentro de las políticas públicas, jerarquizándose al interior de los organigramas estatales y logrando resultados significativos para la posguerra como la duplicación de las camas hospitalarias, la disminución de la mortalidad bruta y la erradicación de algunas endemias regionales como el paludismo.

Ese proceso de construcción de políticas sanitarias implicó ciertas particularidades y, aunque en esos años se consolidó una configuración estatal más intervencionista, el aumento de las atribuciones del Estado central argentino sobre el resto de las jurisdicciones no supuso la eliminación de la heterogeneidad y la fragmentación del sistema público de salud (Belmartino et al., 1991, p.58). Las investigaciones dedicadas a las políticas nacionales constatan que los espacios provinciales mantuvieron una singular importancia en la oferta de servicios y para la década de 1950 los objetivos tendientes a su nacionalización fueron marginados de los planes gubernamentales (Ramacciotti, 2009). Los estudios realizados desde perspectivas provinciales (Hirschegger, 2007-2008; Rodríguez, 2011) también verifican la escasa gravitación del Estado federal en el tratamiento de las problemáticas sanitarias y el mayor protagonismo que tuvieron los estados provinciales en la distribución de la salud durante los gobiernos peronistas, desarrollando incluso políticas innovadoras respecto al accionar nacional (Valobra, 2007). Para mediados de la década de 1950, en algunas jurisdicciones argentinas, el grueso de la asistencia médica de la población recayó, entonces, más sobre las oficinas y los presupuestos locales que sobre los nacionales.

En el marco de esas investigaciones, este trabajo tiene como propósito rastrear cómo se desarrolló la expansión de las intervenciones sanitarias en los espacios provinciales, centrándonos específicamente en quiénes fueron los actores involucrados y de qué modo a lo largo del tiempo se desplegó la provincialización de los servicios de salud. Para ello, en el artículo, analizamos los cambios introducidos en las políticas sanitarias ejecutadas y las interrelaciones entre sus diferentes proveedores en la provincia de Córdoba entre 1930 y 1955, recorte que responde a las coordenadas temporales principales del proceso estudiado.

En este punto resulta evidente que la adopción de una escala provincial para abordar la construcción de las políticas sanitarias no se justifica por su valor agregativo a lo conocido previamente, sino por todo aquello que permite complejizar, revisar y cuestionar respecto a aquellas investigaciones construidas desde el ámbito nacional. En esos términos, la escala provincial permite interpelar los relatos mayores, mostrando discontinuidades y periodizaciones específicas poco visibles desde otras escalas espaciales. Esta aproximación desde un marco provincial hace posible, por lo tanto, analizar problemas generales poniendo en evidencia aspectos poco considerados y revelando las particularidades de los procesos históricos en su despliegue temporal y espacial.

Desde esa perspectiva analítica, nuestro supuesto principal es que en el país se produjeron diversos ritmos en la difusión de las políticas sociales y, durante las primeras décadas del siglo XX, el Estado nacional sufrió numerosos desajustes para extender sus intervenciones sobre 
todo el territorio argentino. En ámbitos provinciales, como el cordobés, la expansión de los servicios de salud se tornó visible recién en el período de entreguerras y, más específicamente, desde 1930. Esto puede comprenderse principalmente a partir de las transformaciones introducidas en los aparatos provinciales, en la atención sanitaria de la población con un incremento gradual, pero constante, de los gastos presupuestarios, de los efectivos estatales que conformaban la burocracia del área y de la infraestructura hospitalaria dependiente de entes públicos (Ortiz Bergia, 2012a). En otras palabras, la descentralización habría sido un rasgo constitutivo del sistema de salud en la Argentina y sus jurisdicciones, como la cordobesa, fueron las responsables centrales de su estructuración.

Ahora bien, aunque las agencias cordobesas fueron las principales proveedoras de los servicios de salud durante el período en estudio, es posible aprehender que el Estado nacional adoptó roles específicos en la conformación de ese sistema de salud. Durante esos años, las agencias nacionales ampliaron sus intervenciones a través de un esquema geográficamente coordinado que distribuía recursos y demanda sanitaria entre los distintos espacios provinciales en el tratamiento de algunas afecciones como las enfermedades psiquiátricas e infectocontagiosas. En otras palabras, aunque en el período se desarrolló un proceso de provincialización de los servicios sociales, esto no se desenvolvió de forma excluyente y las reparticiones federales asumieron funciones específicas.

Es necesario señalar que en investigaciones previas hemos realizado avances en la comprensión del proceso de descentralización de los servicios de salud a través de la exploración de diversos aspectos relativos a las agencias sanitarias cordobesas en el período en estudio. En la trayectoria de la repartición provincial, inicialmente indagamos en el Consejo Provincial de Higiene, tratando de ponderar los cambios atravesados en sus intervenciones y en sus capacidades administrativas en el período 1930-1943. Esto nos permitió constatar la existencia de un lento proceso de construcción de políticas sociales centradas en la jurisdicción cordobesa durante la entreguerras. Las mismas fueron el resultado de un conjunto de factores como la emergencia de tendencias intervencionistas en las esferas estatales, la circulación de lenguajes eugenésicos y la actuación de médicos "notables" al interior del aparato estatal (Ortiz Bergia, 2012a). Este análisis posteriormente se extendió para incluir algunos de los rasgos que adoptó esa agencia durante el período peronista, a través de la descripción de las modificaciones generadas en la infraestructura de salud, el gasto público y los agentes sanitarios dependientes de las oficinas provinciales (Ortiz Bergia, 2012b). Finalmente, esas aproximaciones han sido complementadas con un trabajo dedicado a comprender cómo las transformaciones en el esquema de salud provincial incidieron en las relaciones interjurisdiccionales. Para ello, abordamos las relaciones entre las agencias federales y las locales durante la coyuntura 1943-1946, intentando reconstruir las posiciones que los funcionarios cordobeses adoptaron respecto a la coordinación de las políticas públicas y cómo sus actitudes variaron a lo largo del tiempo (Ortiz Bergia, 2012c). El conjunto de esas publicaciones fue el resultado de una tesis doctoral dedicada al estudio de las políticas sociales en la ciudad de Córdoba entre 1930 y 1955.

En contraposición a esas investigaciones previas, este trabajo busca ponderar cuáles fueron los principales responsables en la estructuración de los servicios de salud gratuitos en la jurisdicción cordobesa y cómo su participación se modificó a lo largo del tiempo. Esto implica superar el análisis del Estado provincial para incluir a otros proveedores como las agencias 
nacionales, municipales y las diversas organizaciones de la sociedad civil. Para abordar ese problema hemos dividido el trabajo en dos partes. En la primera, analizamos la expansión de los servicios existentes en Córdoba y, en la segunda, indagamos el fenómeno de su provincialización, a través de las características que el proceso adoptó y del desenvolvimiento de los distintos actores intervinientes dentro del mismo.

\section{La infraestructura de salud en la provincia de Córdoba}

Las investigaciones sobre historia de la salud suelen destacar que en la ciudad de Buenos Aires, desde comienzo del siglo XX, se fue reduciendo la brecha entre las expectativas de reforma social y la generación de un equipamiento sanitario, producto del intensivo accionar del Estado federal, municipal y de las asociaciones privadas de mutualismo y de beneficencia. Durante el período de entreguerras, dada esa oferta sanitaria existente, se produjeron intensos debates sobre la necesidad de regular y reorganizar ese sistema, y se generó una mayor intervención estatal orientada a ampliar los servicios de salud para integrar a nuevos sectores sociales (Garnino, Persello, 1988; Belmartino, 2005). Pero la trayectoria del sistema de salud porteño difícilmente puede ser generalizada al resto del país e, incluso, es posible constatar que en esa época la mayoría de las jurisdicciones políticas argentinas tenían severas dificultades en la construcción de instrumentos eficaces para el tratamiento de las condiciones de salud de su población. Como manifestación de lo afirmado, en esa coyuntura, el esquema sanitario cordobés se caracterizó por una insuficiente penetración de infraestructura sanitaria, en especial en las zonas más marginales socioeconómicamente como el noroeste. En el caso de Córdoba, en 1930, con 300.000 habitantes, disponía de apenas seis hospitales generales para cubrir la demanda de la ciudad y la proveniente del interior provincial (el Hospital Universitario, el Hospital de Niños de la Sociedad de Beneficencia, el Español y el Italiano de las sociedades de beneficencia de sendas colectividades y el Hospital San Roque bajo el patronato del gobierno provincial). En consecuencia, ese esquema público se manifestaba como un deficiente tendido de dispositivos que no se adecuaban a la dispersión geográfica ni al volumen de su población. Los médicos distaban de lograr "el monopolio del saber y de las prácticas médicas" y debían competir con otras estrategias de acceso a la salud como los curanderos (Moreyra, 2009, p.72-73, 129-130).

Para mediados de 1950 fue evidente la generación de cambios estructurales en ese sistema con el incremento sustancial de los dispositivos públicos al alcance de la sociedad. Esto fue el resultado del accionar concurrente de asociaciones civiles como las tradicionales organizaciones de beneficencia y de los aparatos estatales - nacionales, provinciales y municipales. En esos términos, entre los años 1930 y los gobiernos peronistas, se produjo un incremento sustancial en las camas de internación policlínica y en los servicios ambulatorios gratuitos. Este fenómeno también se visibilizó en el crecimiento de los establecimientos de internación monoclínica de la población, con el aumento de la oferta global de asistencia para el tratamiento de diversas enfermedades como las psiquiátricas, el paludismo, la brucelosis, las venéreas, la lepra y la tuberculosis. Para el caso de la ciudad de Córdoba, capital de la jurisdicción, esas modificaciones en la infraestructura de salud produjeron un mejoramiento de los índices vitales de la población con una caída de la mortalidad infantil, 
de la incidencia de las enfermedades infecciosas en la mortalidad bruta y el aumento de la esperanza de vida al nacer (Celton, 1992). De todos modos, es necesario advertir que para el Estado nacional ese mejoramiento, como ha destacado Karina Ramacciotti (2009, p.100), no estuvo exento de tensiones. En nuestro caso, las críticas a los servicios hospitalarios públicos se dirigieron a los desajustes materiales entre un esquema sanitario en crecimiento y la escasez de dispositivos para asegurar la eficacia de las prestaciones proporcionadas, como el acceso a los medicamentos.

Para ponderar los cambios mencionados previamente, hemos reconstruido estadísticamente la evolución de la infraestructura hospitalaria policlínica pública en el período en estudio. Con esa finalidad, hemos elegido el número de establecimientos y camas de internación hospitalaria como indicadores de este proceso, dado que para la elite médica de la época eran símbolos privilegiados de la modernización sanitaria y de la democratización de la salud (Ramacciotti, 2009, p.100).

Respecto a los datos siguientes, es necesario advertir que las cifras están infravaloradas, pues no están completas y no hemos contabilizado algunos establecimientos y camas gratuitas. ${ }^{1}$ Consideramos, no obstante, que podemos adoptar los guarismos proporcionados como indicadores aproximados. A partir de la Tabla 1 podemos constatar, entonces, las aseveraciones anteriores respecto al incremento producido en las prestaciones sanitarias gratuitas en la jurisdicción cordobesa durante el período en estudio. Así pues, las cifras muestran que entre 1930 y 1955 se generó un aumento sustancial en la oferta de servicios en la provincia de Córdoba y, como manifestación de ello, las camas hospitalarias policlínicas disponibles en forma gratuita se incrementaron en un $226 \%$.

\begin{tabular}{lrrrrr}
$\begin{array}{l}\text { Tabla 1: Establecimientos hospitalarios policlínicos públicos } \\
\text { en la provincia de Córdoba, 1930, }\end{array}$ & $1935,1943,1951$ y 1954 \\
\hline & 1930 & 1935 & 1943 & 1951 & 1954 \\
\hline Establecimientos hospitalarios & 16 & 22 & 32 & 27 & 110 \\
Camas hospitalarias & 1595 & 1741 & 2350 & 2755 & 5197 \\
\hline
\end{tabular}

Fuentes: Córdoba, 1930; Córdoba, 1935; Córdoba, 1943; Argentina, 1952; Argentina 1954, p.20.

Las estadísticas insertadas previamente muestran que ese proceso no fue del todo homogéneo durante toda la etapa y, por el contrario, se desarrolló a velocidades diferentes según los contextos políticos y económicos atravesados por la jurisdicción. Las camas hospitalarias aumentaron un 4\% en el período 1930-1935; un 17\% durante los años 1936-1943; un $11 \%$ en el período $1943-1951$ y un $68 \%$ entre 1952-1954. Estas cifras muestran que el incremento se produjo a partir de la segunda mitad de los años 1930, coincidiendo con el período de recuperación posterior a la crisis económica y con el ascenso al poder local de las administraciones radicales sabattinistas, una tradición partidaria local, identificada en lo social por sus posiciones favorables al intervencionismo estatal. En esos años, sin embargo, aunque la atención de las condiciones sanitarias de la población alcanzó un lugar más preponderante que en las décadas previas, todavía se mantuvo subordinada a otras problemáticas económicas y políticas provinciales (Ortiz Bergia, 2012a). Por el contrario, a comienzos de los años 1950 se generó un verdadero quiebre en las dimensiones de la infraestructura sanitaria cordobesa, 
una jerarquización vinculada a la valorización de la salud como instrumento de progreso económico y social. Dentro del aparato estatal, esto se visibilizó en la participación relativa de la inversión en salud dentro del gasto total, ${ }^{2}$ en la creación de organismos de jerarquía ministerial para tratar esas temáticas y en el crecimiento de la burocracia sanitaria. Así pues, en ese período, la agencia sanitaria provincial se convirtió en Ministerio de Salud Pública y Asistencia Social (1950) y, entre 1943 y 1950, se multiplicó en nueve el personal dependiente del mismo (Córdoba, 1950; Córdoba, 27 ene. 1950; Córdoba, 9 mar. 1950).

Para ponderar más acabadamente ese mejoramiento de la oferta de servicios de salud a disposición de los cordobeses, también podemos tener en cuenta las transformaciones más generales que se introdujeron en el sistema, con la aparición de un mayor número de prestadores privados, a través de la instalación de empresas médicas y de los primeros hospitales pertenecientes a mutualidades obreras. En 1943, en las fuentes oficiales, se hacía referencia a 4 sanatorios privados en la ciudad de Córdoba, con aproximadamente 40 y 30 camas cada uno, y 17 en el resto de la provincia, incluyendo algunos especializados en el tratamiento de la tuberculosis. Para 1951 hemos constatado la existencia de aproximadamente 22 sanatorios privados, solamente en la ciudad de Córdoba (Guía..., 1951) y dos hospitales obreros, el de los ferroviarios y el de los bancarios (Argentina, 1947b, p.692; Inauguran..., 31 mayo 1952; Los servicios..., 10 abr. 1954). Estos nuevos establecimientos y su dispersión en el territorio provincial implicaron la construcción paulatina de una oferta orientada a satisfacer la demanda de los sectores con mayor poder adquisitivo, delimitando también una clientela para los servicios hospitalarios públicos, centrada en los sectores más empobrecidos de la sociedad. ${ }^{3}$ Vale la pena detenerse en ese hecho porque el mismo implicó la cristalización de circuitos institucionales diferenciados socioeconómicamente que se prolongaron a lo largo del siglo XX en la Argentina. De tal modo, en Córdoba, la emergencia del esquema público de salud fue acompañada, en forma paralela, por un sistema privado. Sobre esta cuestión, ha sido Susana Belmartino quien ha analizado más específicamente los cambios que se produjeron en los formatos de las prestaciones médicas y la organización de esos circuitos desde la entreguerras (Belmartino et al., 1988).

Para aprehender el alcance más general del proceso de expansión de los servicios públicos también podemos analizar la evolución de otros dispositivos sanitarios que aumentaron sus prestaciones en esa época como los consultorios médicos, dispensarios, centros de salud y salas de primeros auxilios. Para ello, en la Tabla 2, incluimos el número total de establecimientos sin internación disponibles, según sus proveedores, en 1955. En la Tabla 2 se contabiliza la participación de la provincia, de los municipios y de los particulares en este tipo de servicios.

Es necesario señalar que las prestaciones reseñadas en la Tabla 3 no habían estado necesariamente disponibles a lo largo de las décadas previas, y recién entre los años 1930 y 1940 el municipio cordobés extendió más claramente sus intervenciones. Como expresión de ello, a partir de 1932, comenzaron a erigirse dispensarios seccionales en distintos puntos de la ciudad que permitieron redistribuir los servicios del centro de la urbe hacia los barrios suburbanos. Desde entonces y hasta 1952, se contabilizó la instalación de aproximadamente 21 dispensarios seccionales municipales y se crearon otros servicios rotativos destinados a cubrir la demanda de salud en los barrios periféricos de la ciudad capital (Recorrerá..., 20 ene. 1954; Consultorio..., 6 ene. 1955). 
Tabla 2: Establecimientos de salud sin internación en la provincia de Córdoba, según jurisdicción al 30.6.1955

\begin{tabular}{lc}
\hline Jurisdicción & $\begin{array}{c}\text { Número de } \\
\text { establecimientos }\end{array}$ \\
\hline Ministerio nacional & 8 \\
Otros ministerios nacionales & 1 \\
Provincial & 226 \\
Municipales & 53 \\
Particulares & 91 \\
\hline Total & 379 \\
\hline
\end{tabular}

Fuente: Argentina, 1954, p.667.

En ese período, en la ciudad y en la provincia de Córdoba, el Estado nacional también articuló un sistema de consulta médica más amplio del que había desarrollado en las décadas previas. La existencia de esos servicios ambulatorios, dependientes de la nación, constituye un indicio de la mayor participación de las reparticiones nacionales en las prestaciones de salud recién en la posguerra y de sus intentos por distribuirlas en forma más efectiva en el extenso territorio argentino.

Tabla 3: Infraestructura de servicios nacionales en la provincia de Córdoba, 1943-1955

\begin{tabular}{lll}
\hline Año & Servicio & Ubicación \\
\hline 1943 & Instituto Nacional de Puericultura & Córdoba Capital \\
1943 & Instituto de Cardiología de la Nación & Córdoba Capital \\
1944 & Centro de Inmunización Específica & Córdoba Capital \\
1948 & Instituto Nacional de Hemoterapia & Córdoba Capital \\
1948 & Centro de Vías Respiratorias & Córdoba Capital \\
1948 & Centro de Medicina Preventiva & Córdoba Capital \\
1948 & Centro Serológico - Centro de Higiene Social & Córdoba Capital \\
1949 & Instituto de Reconocimientos Médicos & Córdoba Capital \\
1949 & Dirección Nacional de Ayuda Escolar seccional Córdoba & Córdoba Capital \\
1950 & Dirección de Higiene y Medicina Infantil & Córdoba Capital \\
1950 & Centro de Higiene Materno Infantil & Cruz del Eje \\
1950 & Centro de Salud & Las Varillas \\
1950 & Centro de Higiene Materno Infantil/centro de salud & Villa de María \\
1950 & Servicio de Exámenes Periódicos de la Población & Córdoba Capital \\
1952 & Laboratorio Sanitario de Zona & Córdoba Capital \\
s/a & Centro de Investigaciones Epidemiológicas Regionales & Córdoba Capital \\
\hline
\end{tabular}

Fuentes: Se inaugura..., 7 ago. 1943; La Secretaría..., 24 mayo 1948; El Dr. Carrillo..., 24 sep. 1948; Ha llegado..., 16 oct. 1948; 100.000 vacunas..., 30 sep. 1949; Ha sido..., 20 oct. 1951; La vacunación..., 8 sep. 1944; Symposium..., 22 jul. 1954; Secretaría...,14 nov. 1948; Una campaña..., 1 ene. 1949; Atención..., 23 mar. 1950; Efectiva..., 11 mayo 1950; Argentina, 1954, 1952. 
Por último, para esos años, el Estado provincial también adquirió predominio en el mantenimiento de los servicios de consulta médica. Este proceso se inició durante la primera mitad de la década de 1930, momento en que se crearon, en el interior cordobés, los llamados dispensarios de profilaxis general y primeros auxilios. La finalidad subyacente a estos nuevos establecimientos fue asegurar el acceso a la atención sanitaria asistencial para la población distante de otros servicios públicos (Córdoba, 1992, p.85). En el transcurso de los años 1930 y los 1950, esos dispensarios, ubicados excluyentemente en la campaña, aumentaron nuevamente y su crecimiento no se detuvo en el transcurso de los años. En la posguerra también se incorporó un nuevo formato de establecimiento como la sala de primeros auxilios o puesto sanitario, que implicó un servicio de menor complejidad que el anterior - a cargo de un enfermero en vez de un médico - extendiendo en poco tiempo las prestaciones estatales a pequeñas localidades y pueblos. Si observamos la Tabla 4 es posible aprehender el incremento significativo de ese formato de asistencia dentro del gobierno provincial entre 1930 y 1954.

Tabla 4: Servicios de consulta médica dependientes del gobierno provincial de Córdoba, 1932, 1935, 1943, 1949, 1954

\begin{tabular}{lrrrrr}
\hline & 1932 & 1935 & 1943 & 1949 & 1954 \\
\hline Dispensario con internado & - & - & 35 & - & - \\
Dispensario sin internado & - & 22 & 23 & 137 & 90 \\
Sala de primeros auxilios & - & - & - & - & 78 \\
Dispensario antituberculoso & 4 & 6 & 5 & - & - \\
Dispensario antivenéreo & 14 & 8 & 9 & - & - \\
Dispensario de lactantes/centros materno infantiles & 5 & 7 & 9 & - & 7 \\
Destacamentos sanitarios & - & - & 6 & - & - \\
\hline Total & 23 & 43 & 87 & 137 & 175 \\
\hline
\end{tabular}

Fuentes: Córdoba, s.f.; Córdoba, 1932; Córdoba, nov. 1943; Córdoba, 1 mayo 1949; Argentina, 1954, p.623; Repartición..., 28 jun. 1943.

Es interesante mencionar que la mayor parte de esos servicios provinciales se desplegaron principalmente en los espacios rurales, producto de un viraje en la salud pública que iba desde una preocupación preferentemente orientada a las problemáticas urbanas hacia una mayor consideración por la sanidad rural. Esta cuestión resulta importante para pensar las políticas sociales de la época porque precisamente Adriana Álvarez (2010, p.130) explica que, en el transcurso de las primeras décadas del siglo $\mathrm{XX}$, se comenzó a desarrollar un desplazamiento en la preocupación de las elites médicas estatales argentinas desde las áreas urbanas hacia las rurales. A partir de los años 1930, ese predominio de la sanidad rural sobre la urbana se intensificó, producto de los cambios operados en las condiciones de vida de los espacios urbanos y en la permanencia de enfermedades endémicas y epidémicas en los ámbitos rurales. También, en el caso de Córdoba, María Laura Rodríguez (2010) muestra que en la década de 1930 se produjo el despliegue de intervenciones provinciales más sistemáticas sobre el paludismo en los ámbitos rurales, indicador de la mayor relevancia que comenzó a adquirir el campo sobre la ciudad. 
Durante más de dos décadas, los dispensarios y las salas de primeros auxilios fueron ubicados en la llamada campaña provincial y recién durante los gobiernos peronistas se crearon algunos dispensarios en la ciudad de Córdoba. ${ }^{4}$ El diseño de esas políticas respondió, predominantemente, a la necesidad de adecuar la trama pública asistencial a un contexto sanitario extremadamente disperso, con predominio de población rural, caracterizado por las grandes distancias y las realidades sociales heterogéneas. En particular, los dispensarios y los puestos sanitarios fueron una modalidad de asistencia flexible y adecuada a la realidad rural de buena parte de la jurisdicción. Todos estos fenómenos muestran, en consecuencia, que entre 1930 y 1955, en la provincia de Córdoba, se produjo un incremento sostenido en las prestaciones sanitarias que comenzaron a beneficiar más sistemáticamente a la población de menores ingresos y habitante de los espacios rurales. Estos procesos significaron una mayor disponibilidad de servicios de salud en la que participaron diversos proveedores estatales y civiles.

\section{La provincialización del sistema hospitalario cordobés}

A lo largo de las tres primeras décadas del siglo XX, una de las particularidades del esquema sanitario cordobés había sido la nula participación del gobierno provincial. De ese modo, hasta 1930 las reparticiones provinciales habían reducido sus actividades a la regulación del ejercicio de la medicina y a la intervención esporádica en la atención de las enfermedades infecciosas. También mantenían estrechas relaciones con las asociaciones civiles a través de una política flexible de asignación de subvenciones, subsidios y obras públicas para sustentar el accionar de las organizaciones de beneficencia. Prevalecía, entonces, un sistema prescindente, principalmente orientado por una concepción liberal de las políticas sociales, de políticas sanitarias fuertemente condicionadas por perspectivas epidemiológicas y con actuaciones de carácter más defensivo que preventivo. En ese panorama, hacia mediados de los años 1920, se comenzaron a introducir algunos incipientes cambios (Carbonetti, 2005), aunque la imagen que siguió prevaleciendo fue la de un esquema sanitario con escasa participación provincial. Em 1930, el gobierno bajo su estricto control contaba, en todo el territorio de su jurisdicción, con solo tres dispensarios para niños, tres antituberculosos, un conjunto de establecimientos antivenéreos y un hospital.

Recién durante la década de 1930, es posible apreciar quiebres más significativos en esas tendencias con la paulatina construcción de nuevas intervenciones estatales de las que Córdoba fue solo una de sus expresiones. Al respecto, podemos afirmar que este fue un proceso más generalizado que pareció afectar a diversas provincias a lo largo del país e, incluso, incipientemente a los territorios nacionales. Desde esa perspectiva, los estudios realizados por Susana Belmartino (2007) y, más recientemente, por Susana Piazzesi (2009) sobre las políticas sanitarias en Santa Fe revelan el intenso proceso de elaboración de acciones sociales estatales en esa jurisdicción durante la segunda mitad de la década del 1930. En algunos territorios nacionales, como La Pampa, habría sucedido algo similar. María Silvia Di Liscia (2007) asevera que en esa jurisdicción se incrementó la preocupación estatal por la situación sanitaria de la población a comienzos de los años cuarenta. En Córdoba para esa época, como veremos, también se produjo un viraje en la actitud prescindente 
del Estado provincial respecto a las demandas de salud de la población y se generaron incipientes aumentos en los hospitales dependientes del mismo. Este proceso, además, se manifestó en el incremento de los espacios de consulta médica y en las tareas vinculadas a la medicina preventiva.

Para visibilizar esa gradual provincialización del sistema de salud hemos volcado el número de establecimientos policlínicos existentes en la provincia y de las camas dependientes de los mismos, según su jurisdicción y localización, en distintas fechas entre 1930 y 1954. A partir de la Tabla 5, una de las primeras observaciones que se pueden hacer es la destacada participación de las asociaciones civiles a comienzos del período en estudio. A inicios de esa época, las organizaciones de beneficencia administraban más del 61\% de los servicios hospitalarios. Del resto de la infraestructura disponible solo un 39\% correspondía al Estado, un $15 \%$ al nacional y un reducido $2,5 \%$ al provincial. El Estado provincial, en consecuencia, tenía una muy escasa participación en la resolución de las problemáticas sanitarias. A esto podemos agregar que, en esos años, la política provincial respecto a la oferta hospitalaria se caracterizaba, predominantemente, por subvencionar económicamente la labor desarrollada por asociaciones civiles.

En la etapa siguiente (1935) se desarrollaron algunas novedades en ese esquema con el incremento reducidísimo de la participación provincial: solo el 4\% de la estructura de salud.

Tabla 5: Establecimientos hospitalarios policlínicos públicos en la provincia de Córdoba por dependencia, 1930, 1935, 1943, 1951, 1954*

\begin{tabular}{|c|c|c|c|c|c|c|c|}
\hline & Localización & Nación & Universidad & Provincia & Municipios & Org. Beneficencia & TOTALES \\
\hline & Interior & $240(1)^{* *}$ & & $40(1)$ & & $396(9)$ & $676(11)$ \\
\hline & Capital & & $340(1)$ & & & $579(4)$ & $919(5)$ \\
\hline \multirow[t]{3}{*}{1930} & Total & $240(1)$ & $340(1)$ & $40(1)$ & & 975 (13) & $1.595(16)$ \\
\hline & Interior & $320(1)$ & & $66(1)$ & & $639(16)$ & $1.025(18)$ \\
\hline & Capital & & $350(1)$ & & & $366(3)$ & $716(4)$ \\
\hline \multirow[t]{3}{*}{1935} & Total & $320(1)$ & $350(1)$ & $66(1)$ & & $1.005(19)$ & $1.741(22)$ \\
\hline & Interior & $350(1)$ & & $186(8)$ & $134(4)$ & $611(15)$ & $1.281(28)$ \\
\hline & Capital & & $420(1)$ & $318(1)$ & & $331(2)$ & 1.069 (4) \\
\hline \multirow[t]{3}{*}{1943} & Total & $350(1)$ & $420(1)$ & $504(9)$ & $134(4)$ & $942(17)$ & $2.350(32)$ \\
\hline & Interior & $942(3)$ & & $618(15)$ & $28(1)$ & $67(3)$ & $1.655(22)$ \\
\hline & Capital & & $456(1)$ & $592(2)$ & & $52(2)$ & $1.100(5)$ \\
\hline \multirow[t]{3}{*}{1951} & Total & $942(3)$ & $456(1)$ & $1.210(17)$ & $28(1)$ & $119(5)$ & $2.755(27)$ \\
\hline & Interior & $880(3)$ & & sin datos & & & sin datos \\
\hline & Capital & & & sin datos & & & sin datos \\
\hline $1954^{* * *}$ & Total & $880(3)$ & & $4.317(107)$ & & & $5.197(110)$ \\
\hline
\end{tabular}

\footnotetext{
* Los datos correspondientes a los años 1930 y 1943 han sido previamente publicados (Ortiz Bergia, 2012c).

** Entre paréntesis contabilizamos el número de establecimientos.

*** Se suman las 320 camas en el hospital de Bell Ville en 1946 y las ampliaciones e inauguraciones realizadas para 1951.

Fuentes: Córdoba, 1930; Córdoba, 1935; Córdoba, 1943; Argentina, 1952, p.17, 20; Argentina, 1954, p.7, 623.
} 
En cambio, la nación participaba con un 18\% y las organizaciones de beneficencia con un $58 \%$. Esto implicó cambios apenas perceptibles entre los que podemos mencionar el aumento mínimo de la participación nacional y provincial. Al concluir los gobiernos radicales (1943) se habían generado modificaciones más sustanciales y las organizaciones de beneficencia habían perdido participación relativa, disminuyendo su accionar en un 18\%, pasando a administrar solamente el 40\% de la estructura hospitalaria. El gobierno provincial absorbió esa reducción con una ampliación en su participación que alcanzó al 21\% de las camas de medicina policlínica. A su vez, compartió con la nación (15\%), la Universidad (18\%) y los municipios (6\%) una participación similar en la salud. Durante los gobiernos radicales, por lo tanto, se introdujo una ruptura en la tradicional prescindencia del Estado provincial respecto a las problemáticas sanitarias y crecieron los servicios hospitalarios estatales a costa de la sociedad civil. Estas tendencias evidenciaron el lento viraje de la participación del Estado provincial en la administración hospitalaria y la ruptura de décadas de prescindencia.

Los años que siguieron a 1940 implicaron una etapa más significativa en ese fenómeno de emergencia del Estado provincial como principal proveedor de los servicios de salud públicos. Para 1951 es posible observar que solo el 4\% de la infraestructura hospitalaria estaba administrada por asociaciones de beneficencia. Dentro del 96\% restante se había producido un incremento de la participación nacional con la dirección del 34\% de las camas hospitalarias. ${ }^{5}$ Sin embargo, el principal actor en la administración hospitalaria de clínica general era el gobierno provincial con el 44\% de las prestaciones. En 1954, finalmente, se acentuaron esas tendencias con una ampliación aún mayor de la administración hospitalaria provincial. En esa fecha, el 83\% de las camas de clínica general y cirugía de la jurisdicción eran administradas por el Estado cordobés. En menos de dos décadas, la infraestructura sanitaria había sido estatizada y, fundamentalmente, provincializada.

Esa ampliación de la acción del Estado provincial implicó, simultáneamente, la provincialización de la infraestructura de otras jurisdicciones estatales y entidades civiles. Hacia finales de los años 1940, en la jurisdicción cordobesa, el grueso de los hospitales dependientes de organizaciones civiles y buena parte de los municipales habían sido traspasados al Estado provincial (Ortiz Bergia, 2012d). Entre las organizaciones civiles hemos contabilizado 26 establecimientos de beneficencia intervenidos o donados, entre 1949 y 1955, al Estado provincial. Para 1950, se habían entregado a la provincia 2.500 camas hospitalarias que demandaban anualmente para su atención 25 millones de pesos (Córdoba, 22 dic. 1950). Desde el ámbito nacional se incentivó este proceso y el ministro nacional de salud, Ramón Carrillo, afirmó que los hospitales de beneficencia debían pasar "al patrimonio provincial con la ayuda financiera de la Nación". Su plan consistía en que la estructura hospitalaria asistencial quedara en manos provinciales, mientras la atención preventiva de la salud sería de responsabilidad nacional (Inaugurose..., 3 mayo 1950).

En el caso de los municipios, varios de ellos también transfirieron su infraestructura hospitalaria al Estado provincial como el hospital Rawson de infectocontagiosos, el hospital y el asilo municipal de Altagracia, el hospital San Isidro de Oncativo, el de La Falda, el dispensario de General Deheza y el de Sampacho (Córdoba, 16 dic. 1949; Córdoba 23 ago. 1955; Córdoba, 28-30 dic. 1953). Poco tiempo después un funcionario cordobés informaba a las autoridades municipales que la provincialización de la infraestructura hospitalaria era 
"a los fines de aliviar los presupuestos municipales en las partidas que tenían destinadas a la medicina asistencial, el gobierno se ha hecho cargo prácticamente, de todos los servicios asistenciales de la provincia." En contraprestación, las autoridades provinciales solicitaban que los municipios cooperaran en la acción social como el cuidado de los ancianos y los niños (Continúa..., 20 nov. 1953).

Es posible afirmar que para los años 1950 estos fenómenos permitieron centralizar alrededor del Estado provincial buena parte de la oferta hospitalaria de la jurisdicción. Ahora bien, ese proceso de provincialización también tuvo sus límites. El Estado nacional mantuvo cierto protagonismo en la provisión de los servicios de salud y la provincia no logró incorporar toda la oferta pública. Ese mantenimiento de la participación del Estado nacional en el esquema cordobés estuvo fundamentalmente vinculado a los hospitales monoclínicos nacionales que en 1954 concentraban el 78\% de las camas de ese tipo existentes en la jurisdicción. Estos servicios se distribuían entre el Sanatorio Puente de San Francisco del Chañar para el tratamiento de la lepra, el Centro Tisiológico Nacional conformado por el Sanatorio Santa María y el Domingo Funes, y la Colonia de Alienados Vidal Abal de la localidad de Oliva. Para ser más específicos, según las estadísticas oficiales, en 1954, la nación administraba 5.537 camas de las utilizadas en la provincia de Córdoba (4.148 psiquiátricas, 1.253 tisiológicas y 136 de leprología). Esta última, en cambio, informaba que en 1955 estaba administrando siete establecimientos monovalentes con 1.522 camas (Argentina, 1954, p.35, 39; Córdoba, 1 mayo 1955).

Empero, es necesario relativizar esa gravitación del Estado nacional en la provincia porque su posición geográfica y ambiental hizo que en esta se produjera una mayor concentración de establecimientos monovalentes nacionales, psiquiátricos y tisiológicos, que en el resto del país. Siguiendo las estadísticas de 1946, en el territorio cordobés se radicaba el 26\% de las camas de dependencia nacional de psiquiatría de todo el país (2.500 de 9.623), pero solo el 9\% de la población total según el censo nacional de 1947. Para 1954 las estadísticas no eran del todo diferentes, aunque la infraestructura psiquiátrica nacional había crecido en un $84 \%$, la provincia seguía absorbiendo el $23 \%$ de las camas nacionales en un solo establecimiento, la Colonia Vidal Abal de la localidad de Oliva (4.148 de 17.695). Otro tanto puede decirse del tratamiento de la tuberculosis. En 1946, entre el Sanatorio Santa María y el Sanatorio Domingo Funes, localizados ambos en las sierras cordobesas, absorbían el 83\% de las camas nacionales dedicadas a la tuberculosis (1.470 de 1.770). Para 1954, cuando las camas nacionales dedicadas a esta afección se habían incrementado en un 45\%, la provincia todavía concentraba el 49\% de las camas existentes (1.253 de 2.574) (Argentina, 1952, p.16; Argentina, 1954, p.35). Falta afirmar que esa infraestructura prestaba sus servicios a todo el territorio nacional y no únicamente a los cordobeses como muestran las investigaciones dedicadas a analizar las políticas de tratamiento de la tuberculosis durante la primera mitad del siglo XX en el país (Carbonetti, 1998; Armus, 2007). Un funcionario nacional en Córdoba explicaba esta situación: "Es digno de comentario que, por tratarse de Córdoba, de una provincia tributaria de todo el país, por su ubicación geográfica, por sus vías de comunicación, por las bondades de su clima y por la capacidad e importancia de sus establecimientos nacionales asistenciales, soporta el peso de la gran mayoría de enfermos de tuberculosis, de alineados y de lepra, que son enviados desde todos los rumbos para su internación y tratamiento" (Efectiva..., 11 mayo 1950). 
Esa participación del Estado nacional en Córdoba contrasta y, a su vez, explica su ausencia en otras provincias. Para la misma época, Ivana Hirschegger (2010, p.106) reconoce que en Mendoza la actuación del Estado nacional no fue significativa respecto a la provincia e identifica la prevención contra la tuberculosis como su única política sistemática en la jurisdicción. Esto permite comprender que el Estado nacional actuó en los espacios provinciales a partir de un esquema de salud en el que determinadas zonas funcionaban como espacios de internación mientras en otras solo se ejecutaban acciones preventivas. Esto se constata con la comprobación de que las admisiones a esos grandes hospitales monovalentes escapaban a los burócratas nacionales radicados en Córdoba. La colonia psiquiátrica de Oliva, el Centro Tisiológico de Punilla y el Sanatorio Puente de Leprología, en San Francisco del Chañar, dependían directamente de la Comisión Nacional de Asilos y Hospitales Regionales y, posteriormente, del Ministerio Nacional de Salud Pública a través del Consejo de Grandes Luchas Sanitarias.

En el caso de Córdoba es posible constatar, entonces, que el Estado nacional no tuvo un papel menor en la jurisdicción y, por el contrario, constituyó un agente importante, asegurando los principales espacios de internación para los enfermos psiquiátricos, tisiológicos y de leprología. Junto a estos debemos incluir algunos policlínicos nacionales con un gran número de camas como el hospital Central de Bell Ville y el Regional de Río Cuarto. De todos modos, esa infraestructura nacional, al no estar solamente orientada a los enfermos de Córdoba, no invalida que el proceso más importante desarrollado en esos años fue la provincialización del esquema de salud y fundamentalmente de la atención de clínica general.

\section{Consideraciones finales}

En la primera mitad del siglo XX, se produjo una ampliación de los servicios de salud públicos existentes en la Argentina y la población alcanzó un mayor y mejor acceso a los mismos. Sin embargo, a lo largo del país esto se produjo de manera poco uniforme y existieron diversos ritmos, alcances y especificidades geográficas. Para ahondar en esas particularidades regionales, en este trabajo analizamos la trayectoria de las políticas sanitarias en Córdoba, en el segundo cuarto del siglo XX, relevando la participación de los diferentes proveedores que actuaron en su estructuración como los gobiernos municipal, provincial y nacional y las organizaciones de beneficencia.

Respecto a esta temática, en artículos anteriores analizamos cómo el Estado provincial en el período de entreguerras atravesó importantes transformaciones en el área sanitaria con un incremento de sus capacidades administrativas, cambios en sus intervenciones y aumentos en su infraestructura de atención (Ortiz Bergia, 2012a). Durante esa etapa Córdoba no habría sido un caso aislado en la Argentina y otras provincias, como Santa Fe, también habrían tenido un desenvolvimiento similar (Belmartino, 2007; Piazzesi, 2009).

La constatación de esas transformaciones en las reparticiones cordobesas, asimismo, nos impulsó a interrogarnos sobre el modo en que las mismas afectaron el funcionamiento de la agencia sanitaria provincial en los años subsiguientes. Más precisamente, exploramos cómo las herencias institucionales producidas en los años 1930 incidieron en las posturas locales respecto a las propuestas de coordinación y centralización sanitaria emanadas de las oficinas 
nacionales en la década de 1940 (1943-1946). Esto último nos permitió visibilizar que el proceso de centralización de las políticas sociales en el país estuvo fuertemente modelado por las estructuras políticas y burocráticas provinciales, las que condicionaron sus posibilidades, límites y resultados (Ortiz Bergia, 2012c).

A la vista de esas conclusiones, en esta última aproximación nos propusimos comprender cómo, en el amplio territorio cordobés, ese esquema estructurado provincialmente se insertó en un sistema de salud público heterogéneo del que también participaban otros oferentes como el Estado nacional, la municipalidad y las organizaciones de beneficencia. Para ello, nos cuestionamos, más precisamente, sobre el modo en que se modificó la oferta de servicios sanitarios entre 1930 y 1955 y cómo en ese proceso varió la actuación de los diferentes proveedores existentes en la jurisdicción.

En el análisis pudimos verificar que en provincias como Córdoba la expansión de los servicios de salud se desarrolló principalmente a partir del período de entreguerras y, entre 1930 y 1955, se exteriorizó en el aumento de las camas de internación policlínicas y monoclínicas y en las prestaciones ambulatorias gratuitas. Estos procesos, junto a la multiplicación paralela de los servicios privados, implicaron una ampliación de los instrumentos de atención disponibles para la población en la ciudad y en la provincia de Córdoba en forma gratuita y arancelada. En lo que se refiere específicamente a los servicios públicos, su expansión permitió una mayor distribución de servicios de salud para la población de menor poder adquisitivo y, en especial, para las personas aisladas de otras prestaciones. En esos términos, un rasgo importante de las políticas impulsadas en ese período fue el intento por distribuir la oferta sanitaria y orientar la infraestructura pública hacia los espacios rurales.

Es posible señalar, además, que ese incremento de las prestaciones dependió fundamentalmente de proveedores locales antes que nacionales, como las organizaciones de beneficencia, los gobiernos provinciales y los municipales. Incluso, una mirada más focalizada permite comprender que en esos años se desplegó una provincialización del sistema antes que una nacionalización. Esto significó que el Estado cordobés incrementó su participación en la atención de las demandas de la población y, a su vez, estatizó la infraestructura de salud perteneciente a otras jurisdicciones y asociaciones civiles. En esa coyuntura, si bien la nación tuvo un papel relevante en la provisión de los servicios de salud, su acción se orientó en forma predominante al tratamiento de algunas enfermedades y tuvo un rol menor al desplegado por las reparticiones provinciales. Es importante detenerse en este último fenómeno porque, en definitiva, muestra que la descentralización de los servicios de salud fue un rasgo constitutivo del sistema sanitario argentino desde su misma conformación y, simultáneamente, permite comprender su extrema heterogeneidad a lo largo del territorio nacional.

\section{AGRADECIMIENTOS}

Agradezco a Karina Ramacciotti su generosa lectura de este trabajo y las sugerencias realizadas al mismo. Hago extensivo mi reconocimiento a los evaluadores anónimos y a los editores de la revista por sus comentarios.

\section{NOTAS}

${ }^{1}$ En las estadísticas relevadas no está contemplado el hospital Córdoba/Eva Perón, inaugurado en 1950,
y otro tanto puede decirse de otros establecimientos sanitarios nacionales en el territorio cordobés. Las
estadísticas tampoco dan cuenta de las camas de internación en los dispensarios, distribuidos en la campaña 
provincial. Para 1943, hemos calculado que las camas de clínica general de este tipo eran 164 en toda la provincia, estas habrían incrementado un 33\% las 504 camas hospitalarias pertenecientes a la jurisdicción provincial para esa fecha (Córdoba, nov. 1943).

${ }^{2}$ El gasto sectorial en salud pasó de representar el 8\% del presupuesto provincial en 1943 a insumir en 1954 el 19\% del gasto total. Esto implicó un porcentaje de aumento en valores reales del 398\% (Argentina, s.f.; Córdoba, 1943, p.429; Córdoba, 29 jun. 1955).

${ }^{3}$ Incluso, durante los gobiernos peronistas todavía se mencionó ocasionalmente al "certificado de pobre" como un mecanismo de acceso a los servicios de salud, aunque probablemente sólo funcionó circunstancialmente.

${ }^{4}$ Lo Dispensario de Rivera Indarte, en 1947, y lo de Villa Belgrano, en 1951 (Inaugurose..., 29 abr. 1947; Se inauguró..., 29 oct. 1951).

${ }^{5}$ De todos modos, estas cifras diferían mucho de las planificadas en 1947, en el Plan Analítico de Salud Pública que preveía para Córdoba 174 servicios nuevos (Argentina, 1947a).

\section{REFERENCIAS}

100.000 VACUNAS...

100.000 vacunas se distribuyeron ayer en esta, según la Dirección de S. Pública. La Voz del Interior, p.11. 30 sep. 1949.

ÁLVAREZ, Adriana.

Entre muerte y mosquitos: el regreso de las plagas en la Argentina (siglos XIX y XX). Buenos Aires: Biblos. 2010.

\section{ARGENTINA.}

Ministerio de Asistencia Social y Salud Pública. Síntesis estadística de la labor realizada por los establecimientos médico-asistenciales de dependencia nacional y provincial: año 1954. Buenos Aires. 1954.

ARGENTINA.

Ministerio de Salud Pública. Memoria correspondiente al período junio 1946 a mayo 1952. Buenos Aires: Departamento de Talleres Gráficos. 1952.

\section{ARGENTINA.}

Secretaría de Salud Pública de la Nación. Plan Analítico de Salud Pública. t.4. Gráficos. Buenos Aires. 1947a.

\section{ARGENTINA.}

Secretaría de Trabajo y Previsión de la Nación. Memoria año 1947. Buenos Aires. 1947b.

\section{ARGENTINA.}

Instituto Nacional de Estadísticas y Censos. Serie histórica del Índice de Precios al Consumidor en la Gran Buenos Aires. Disponible en: http://www. indec.mecon.ar/informacion-de-archivo.asp. Acceso en: 15 mayo 2012. s.f.

ARMUS, Diego.

La ciudad impura: salud, tuberculosis y cultura en Buenos Aires, 1870-1950. Buenos Aires: Edhasa. 2007.

\section{ATENCIÓN...}

Atención médica y odontológica gratuita van a tener los niños. Los Principios, p.3. 23 mar. 1950.

\section{BELMARTINO, Susana.}

Coyuntura crítica y cambio institucional en salud: Argentina en los años 40. Salud Colectiva, v.3, n.2, p.177-202. 2007.

BELMARTINO, Susana.

La atención médica argentina en el siglo XX: instituciones y procesos. Buenos Aires: Siglo XXI Editores. 2005.

BELMARTINO, Susana et al.

Fundamentos históricos de la construcción de relaciones de poder en el sector salud: Argentina 1940-1960. Buenos Aires: Organización Panamericana de la Salud. 1991.

BELMARTINO, Susana et al.

Corporación médica y poder en salud: Argentina, 1920-1945. Rosario: Centro de Estudios Sanitarios y Sociales. 1988.

CARBONETTI, Adrián.

La conformación del sistema sanitario de la Argentina: el caso de la Provincia de Córdoba, 1880-1926. Dynamis, v.25, p.87-116. 2005.

CARBONETTI, Adrián.

Enfermedad y sociedad: la tuberculosis en la ciudad de Córdoba, 1906-1947. Córdoba: Municipalidad de Córdoba. 1998.

CELTON, Dora E.

La mortalidad en la ciudad de Córdoba

(Argentina) entre 1869 y 1990. Boletín de la Asociación de Demografía Histórica, v.10, n.1, p.31-57. 1992.

\section{CONSULTORIO...}

Consultorio de la comuna. Los Principios, p.2. 6 ene. 1955. 
CONTINÚA...

Continúa su labor en esta el I. C. de

Municipalidades. Los Principios, p.6. 20 nov. 1953.

\section{CÓRDOBA.}

Secretaría Técnica Parlamentaria, Cámara de Diputados. Mensajes a la Legislatura acerca del estado de la Provincia: gobernador Pedro J. Frías, 1933-1935. Córdoba. 1992.

\section{CÓRDOBA.}

Ministerio de Salud Pública y Asistencia Social. Decretos de salud pública 4326 D. Serie Decretos, 1955, t.58 (Archivo de Gobierno de la Provincia de Córdoba). 23 ago. 1955.

\section{CÓRDOBA.}

Cámara de Diputados. Diario de Sesiones de la Cámara de Diputados de la Provincia de Córdoba. Córdoba. p.207-208. 29 jun. 1955.

\section{CÓRDOBA.}

Cámara de Senadores. Diario de Sesiones de la Cámara de Senadores de la Provincia de Córdoba. Córdoba. p.69. 1 mayo 1955.

CÓRDOBA.

Cámara de Senadores. Diario de Sesiones de la Cámara de Senadores de la Provincia de Córdoba. Córdoba. p.1446. 28-30 dic. 1953.

\section{CÓRDOBA.}

Cámara de Diputados. Diario de Sesiones de la Cámara de Diputados de la Provincia de Córdoba. Córdoba. p.2174-2175. 22 dic. 1950.

\section{CÓRDOBA.}

Ministerio de Salud Pública y Asistencia Social. Decretos de salud pública 8644 F. Serie Decretos, 1950, t.52 (Archivo de Gobierno de la Provincia de Córdoba). 9 mar. 1950.

\section{CÓRDOBA.}

Ministerio de Salud Pública y Asistencia Social. Decretos de salud pública 7604 F, 7605 F, 7606 F. Serie Decretos, 1950, t.52 (Archivo de Gobierno de la Provincia de Córdoba). 27 ene. 1950.

\section{CÓRDOBA.}

Ley 4244, 29 ago. 1950. Ley de ministerios. Compilaciones de Leyes de la Provincia de Córdoba, p.18. 1950.

\section{CÓRDOBA.}

Ministerio de Salud Pública y Asistencia Social. Decretos de salud pública 6284 F. Serie Decretos, 1949 (Archivo de Gobierno de la Provincia de Córdoba). 16 dic. 1949.

CÓRDOBA.

Cámara de Senadores. Diario de Sesiones de la Cámara de Senadores de la Provincia de Córdoba. Córdoba. p.193. 1 mayo 1949.
CÓRDOBA.

Dirección General de Estadísticas. Morbilidad. Movimiento de los establecimientos oficiales provinciales de Asistencia Médica. Septiembre de 1943. Revista de la Dirección General de Estadística, n.40, p.14-15. nov. 1943.

CÓRDOBA.

Dirección General de Estadísticas. Anuario

Estadístico de la Provincia de Córdoba. Córdoba. 1943.

CÓRDOBA.

Dirección General de Estadísticas. Anuario Estadístico de la Provincia de Córdoba. Córdoba. 1935.

\section{CÓRDOBA.}

Ley 3509, 12 mar. 1932. Ley de presupuesto. Compilaciones de Leyes de la Provincia de Córdoba, p.19-25. 1932.

\section{CÓRDOBA.}

Dirección General de Estadísticas. Anuario Estadístico de la Provincia de Córdoba. Córdoba. 1930.

\section{CÓRDOBA}

Consejo de Higiene. Consejo de Higiene: 19321935. Córdoba: Ompré \& Cía. s.f.

DI LISCIA, María Silvia.

Dificultades y desvelos de un Estado interventor: instituciones, salud y sociedad en el interior argentino, La Pampa, 1930-1946. Anuario del Instituto de Estudios Histórico-Sociales, n.22, p.93123. 2007.

\section{EFECTIVA...}

Efectiva labor há realizado en Córdoba el Ministerio de Salud Pública de la Nación. Los Principios, p.4. 11 mayo 1950.

EL DR. CARRILLO...

El Dr. Carrillo visitará hoy algunos institutos médicos. La Voz del Interior, p.7. 24 sep. 1948.

GARNINO, María Isabel; PERSELLO, Ana

Virginia.

La reformulación del mercado de atención médica, Buenos Aires, 1920-1940. Anuario de Rosario, n.13, p.343-367. 1988.

GUÍA...

Guía Oficial de Córdoba. Córdoba. 1951.

HA LLEGADO...

Ha llegado a esta el grupo móvil de la Direc. de Hemoterapia. La Voz del Interior, p.7. 16 oct. 1948.

HA SIDO...

Ha sido designado nuevo director de exámenes periódicos. La Voz del Interior, p.8. 20 oct. 1951. 
HIRSCHEGGER, Ivana.

Alcance territorial de las políticas públicas peronistas en la provincia de Mendoza (Argentina), 1946-1955. Apuntes, n.66, p.83-115. 2010.

HIRSCHEGGER, Ivana.

La medicina asistencial, sanitaria y social peronista: discursos, acciones y logros: el caso de San Rafael, Mendoza (1949-1952). Revista de Historia Americana y Argentina, n.42-43, p.63-101. 2007-2008.

INAUGURAN...

Inauguran un policlínico. Los Principios, p.2. 31 mayo 1952.

INAUGUROSE...

Inaugurose la C. de coordinación sanitaria.

Los Principios, p.2. 3 mayo 1950.

INAUGUROSE...

Inaugurose un dispensario en Villa R. Indarte.

La Voz del Interior, p.9. 29 abr. 1947.

LA SECRETARÍA...

La Secretaría de Salud Pública de la Nación creará en esta un Centro de Medicina Preventiva.

La Voz del Interior, p.9. 24 mayo 1948.

LA VACUNACIÓN...

La vacunación antidiftérica y antivariólica.

Los Principios, p.4. 8 sep. 1944.

LOS SERVICIOS...

Los servicios sociales para bancarios. Los

Principios, p.2. 10 abr. 1954.

MOREYRA, Beatriz.

Cuestión social y políticas sociales en la Argentina: la modernidad periférica, Córdoba, 1900-1930. Bernal: Universidad Nacional de Quilmes. 2009.

ORTIZ BERGIA, María José.

La salud pública en transformación: la

estructuración de políticas sanitarias en CórdobaArgentina, 1930-1943. Asclepio, v.64, n.1, p.121-146. 2012a.

ORTIZ BERGIA, María José.

La democratización de la salud: las políticas sanitarias durante los gobiernos peronistas en Córdoba (1943-1955). Revista de la Facultad de Ciencias Médica, v.64, n.4, p.228-235. 2012b.

ORTIZ BERGIA, María José.

Provincia y Nación en el proceso de construcción de un Estado centralizado: políticas sanitarias en Córdoba (1943-1955). Quinto Sol, n.1, v.16, p.133-155. 2012c.

ORTIZ BERGIA, María José.

Asistencia benéfica y gobiernos peronistas en Córdoba. In: Biernat, Carolina; Ramacciotti, Karina (Ed.). Políticas sociales, entre demandas y resistencias: Argentina, 1930-1970. Buenos Aires: Biblos. p.67-96. 2012d.

PIAZZESI, Susana.

Conservadores en provincia: el iriondismo santafesino, 1937-1943. Santa Fe: Ediciones UNL. 2009.

RAMACCIOTTI, Karina.

La política sanitaria del peronismo. Buenos Aires: Biblos. 2009.

RECORRERÁ...

Recorrerá los barrios un ómnibus sanitario. $\mathrm{La}$ Voz del Interior, p.4. 20 ene. 1954.

REPARTICIÓN...

Repartición: Departamento Provincial de Higiene. Serie Gobierno, t.1944, t.4, fs.61r87r. (Archivo de Gobierno de la Provincia de Córdoba). 28 jun. 1943.

RODRÍGUEZ, María Laura.

Políticas sanitarias en el interior nacional: el caso de la provincia de Córdoba entre la intervención militar y el primer peronismo (1943-1955). Tesis (Doctorado) - Centro de Estudios Avanzados, Universidad Nacional de Córdoba, Córdoba. 2011.

RODRÍGUEZ, María Laura.

La Campaña Sanitaria Antipalúdica de 1945: un panorama de transición en la región noroeste de Córdoba, Argentina. Trabajo presentado en 4. Taller de Historia Social de la Salud y la Enfermedad, Tucumán. 2010.

SECRETARÍA...

Secretaría de Salud Pública. Dirección de Tuberculosis. Los Principios, p.9. 14 nov. 1948.

SE INAUGURA...

Se inaugura hoy el Dispensario Nacional Cardiólogico de Córdoba. La Voz del Interior, p.6. 7 ago. 1943.

SE INAUGURÓ...

Se inauguró un dispensario en Villa Belgrano. Los Principios, p.2. 29 oct. 1951.

SYMPOSIUM...

Symposium sobre cuidado que requiere el prematuro. Los Principios, p.2. 22 jul. 1954.

UNA CAMPAÑA...

Una campaña interesante. Los Principios, p.4. 1 ene. 1949.

VALOBRA, Adriana.

Un desafío a la justicia social peronista: la hidatidosis en la provincia de Buenos Aires, 1946-1952. História, Ciências, Saúde-Manguinhos, v.14, n.4, p.1357-1375. 2007. 